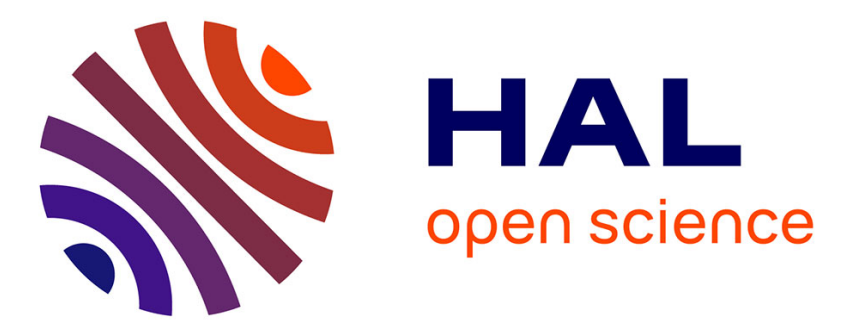

\title{
Croissance et développement du Pachyrhizus erosus Urban. II. Effets de l'acide gibbérellique et du chlorure de chlorocholine sur la composition glucidique du tubercule
}

Claude Zinsou, Gérard Vansuyt, Alfred Venthou-Dumaine, Camille Constant

\section{To cite this version:}

Claude Zinsou, Gérard Vansuyt, Alfred Venthou-Dumaine, Camille Constant. Croissance et développement du Pachyrhizus erosus Urban. II. Effets de l'acide gibbérellique et du chlorure de chlorocholine sur la composition glucidique du tubercule. Agronomie, 1987, 7 (10), pp.821-825. hal00884958

\section{HAL Id: hal-00884958 \\ https://hal.science/hal-00884958}

Submitted on 1 Jan 1987

HAL is a multi-disciplinary open access archive for the deposit and dissemination of scientific research documents, whether they are published or not. The documents may come from teaching and research institutions in France or abroad, or from public or private research centers.
L'archive ouverte pluridisciplinaire HAL, est destinée au dépôt et à la diffusion de documents scientifiques de niveau recherche, publiés ou non, émanant des établissements d'enseignement et de recherche français ou étrangers, des laboratoires publics ou privés. 


\title{
Croissance et développement du Pachyrhizus erosus Urban. II. Effets de l'acide gibbérelli- que et du chlorure de chlorocholine sur la com- position glucidique du tubercule
}

\author{
Claude ZINSOU, Gérard VANSUYT \& Alfred VENTHOU-DUMAINE \\ avec la collaboration technique de Camille CONSTANT
}

I.N.R.A., Laboratoire de Physiologie et Biochimie végétales, Centre de Recherches agronomiques des Antilles et de la Guyane, B.P. 1232, F 97184 Pointe-à-Pitre Cedex (F.W.I., Guadeloupe)

\begin{abstract}
Les effets de l'acide gibbérellique (GA3) et de chlorure de chlorocholine (CCC) ont été étudiés sur la composition glucidique du tubercule du dolique tubéreux, Pachyrhizus erosus (Urban), au cours de son développement. GA3 augmente la teneur du tubercule en sucres solubles totaux alors que CCC l'abaisse mais l'effet de ce dernier n'affecte que les sucres réducteurs. Ces facteurs provoquent dans les tubercules des plantes traitées l'accumulation du saccharose dont le niveau se situe autour de 7 p. 100 alors que ce disaccharide disparaît du tubercule des plantes témoins dès la formation des premières gousses. GA3 stimule la synthèse de l'amidon et son stockage dès l'application. L'effet du CCC est perçu plus tard au moment du remplissage des gousses car il inhibe la chute de la teneur en amidon constatée dans les tubercules des plantes témoins. L'accumulation du saccharose et de l'amidon chez le Pachyrhizus, provoquée par ces deux facteurs de croissance qui ont habituellement des effets contraires chez les plantes à tubercules, est discutée.
\end{abstract}

Mots clés additionnels : Sucres solubles, amidon, accumulation, légumineuse tropicale, dolique tubéreux. chlorocholine chloride on carbohydrate composition of the developing tuberous root.

The effects of gibberellic acid (GA3) and chlorocholine chloride $(\mathrm{CCC})$ on carbohydrate comnosition of tuberous root of yam bean, Pachyrhizus erosus (Urban) were investigated during its development under shortday conditions. Soluble carbohydrate level was increased by GA3 but reduced by CCC, only the reducing sugars being affected in the latter case. Sucrose, which disappeared from the tuber at the beginning of pod setting in the control plants, was maintained by application of each of the two growth regulators. CCC and GA3 both increased starch content in the tuberous root. GA3 promoted early starch synthesis and storage at the same time as sucrose accumulation. The effect of $\mathrm{CCC}$ was observed only at pod bulking, when it inhibited the starch content decrease seen in control plants. The sucrose and starch increases brought about by the two growth factors, which usually have opposite effects on tuber plants, are discussed.

Additional key words : Soluble carbohydrates, starch, accumulation, tropical legume, yam bean.

\section{INTRODUCTION}

L'application des substances de croissance, comme l'acide gibbérellique (GA) et le chlorure de chlorocholine $(\mathrm{CCC})$, au cours de la croissance de $P$. erosus révèle la possibilité de modifier l'importance relative des organes de réserve, l'un par rapport à l'autre. GA favorise le développement du tubercule et améliore le rendement alors que le $\mathrm{CCC}$ oriente vers la production de graines (ZINSOU et al., 1987).

L'intérêt présenté par le tubercule pour l'alimentation humaine et animale a conduit à la détermination de sa composition glucidique et azotée (MARTA EVANS et al., 1977). BABE (cité par MARTINEZ, 1959) trouve dans le tubercule frais 84,7 p. 100 d'eau, 1,7 p. 100 de glucose, 10,72 p. 100 d'amidon (soit respectivement 
11 p. 100 et 70 p. 100 de la matière sèche). L'étude de la composition du tubercule à maturité, entreprise au laboratoire par TREBOS (1982), a donné sur la base de la matière sèche une répartition différente : 8,3 p. 100 pour le glucose, 13,8 p. 100 pour le fructose, 9 p. 100 pour le saccharose, 15,2 p. 100 pour l'amidon. Des analyses ultérieures ont montré que cette composition peut varier dans de larges limites en fonction des conditions climatiques et des pratiques culturales. Il demeure cependant que la teneur en amidon ne dépasse jamais 20 p. 100 , ce qui peut expliquer sa consommation à l'état cru dans de nombreuses régions comme en Amérique centrale (SCHROEDER, 1967) et en Asie (SRIVASTAVA et al., 1973).

Les effets importants de l'acide gibbérellique et du chlorure de chlorocholine (ZINSOU et al., 1987) sur les organes de réserve du dolique tubéreux laissent présager de profondes modifications susceptibles d'être induites par ces facteurs de croissance au niveau de la composition glucidique. Cet article rapporte les résultats obtenus.

\section{MATÉRIEL ET MÉTHODES}

\section{A. Matériel}

Le matériel végétal utilisé est le Pachyrhizus erosus Urban (yam bean ou dolique tubéreux), légumineuse tropicale tubérifère, originaire du Mexique. Ses graines ont été généreusement fournies par le Dr STEELE de l'I.I.T.A. d'Ibadan (Nigeria) et multipliées sur place.

La production du matériel végétal, la fertilisation et l'application de l'acide gibbérellique aux doses de 50 et 200 ppm (GA 50 et GA 200) et du chlorure de chlorocholine à la dose de 1000 ppm (CCC 1 000) ont été réalisées dans les conditions déjà décrites (ZINSOU et al., 1987).

\section{B. Méthodes}

\section{Préparation des échantillons}

Les tubercules de 5 plantes tirées au hasard sont récoltés, lavés, coupés en morceaux et séchés à $80^{\circ} \mathrm{C}$ à l'étuve pendant $48 \mathrm{~h}$. Le matériel est ensuite broyé et tamisé pour avoir une poudre de granulométrie inférieure à $0,4 \mathrm{~nm}$.

\section{Extraction des glucides}

La quantité de poudre à analyser, comprise entre 1 et $2 \mathrm{~g}$, est extraite à chaud 2 fois par $50 \mathrm{ml}$ du mélange éthanol/eau : $80 / 20$ et rincée 2 fois à froid suivant la méthode de CERNING-BEROARD (1975). L'extrait est évaporé sous vide, repris par de l'eau dans une fiole de $100 \mathrm{ml}$ à laquelle on ajoute, pour précipiter les protéines, $1 \mathrm{ml}$ du réactif de Carrez I (23,8 g d'acétate de zinc et $3 \mathrm{~g}$ d'acide acétique glacial dissous dans $100 \mathrm{ml} \mathrm{d}$ 'eau) et $1 \mathrm{ml}$ de Carrez II $(10,5 \mathrm{~g}$ de ferricyanure de potassium dissous dans $100 \mathrm{ml}$ d'eau). La fiole complétée à $100 \mathrm{ml}$ avec de l'eau est agitée et la solution filtrée. Le filtrat est utilisé pour la détermination du glucose, fructose, saccharose et des sucres solubles totaux.

L'amidon est extrait suivant la technique de THIVEND et al. (1965). Le culot, après avoir été repris par 20 à $25 \mathrm{ml}$ dans un bécher de $100 \mathrm{ml}$, subit un $1^{\text {er }}$ empesage en le portant à l'ébullition et en l'y maintenant pendant $3 \mathrm{mn}$, puis un autoclavage à $120^{\circ} \mathrm{C}$ pendant $1 \mathrm{~h}$. La suspension, maintenue à $55^{\circ} \mathrm{C}$, est additionnée de $2,5 \mathrm{ml}$ de solution tampon acétique (120 $\mathrm{ml}$ d'acide acétique cristallisable et $164 \mathrm{~g}$ d'acétate de sodium anhydre/1), $0,5 \mathrm{ml}$ de solution de merthiolate de sodium $(10 \mathrm{~g} / \mathrm{l})$ pour éviter le développement des micro-organismes et ajustée enfin à $(45 \pm 1) \mathrm{ml}$. La suspension est ensuite placée dans un bain-marie agitateur, réglé à $60^{\circ} \mathrm{C}$, et additionnée de $5 \mathrm{ml}$ de solution de glucoamylase (E.C. 3.2.1.3) à $30 \mathrm{UI} / \mathrm{ml}$. L'hydrolyse enzymatique s'effectue pendant $2 \mathrm{~h}$. La solution est ensuite centrifugée et le culot lavé 2 fois avec $50 \mathrm{ml}$ d'eau distillée. Les surnageants sont réunis dans une fiole jaugée qui est ajustée à $250 \mathrm{ml}$.

\section{Dosage des glucides}

Les sucres solubles totaux sont dosés en milieu sulfurique à l'orcinol à l'aide d'un autoanalyseur Technicon (TOLLIER \& ROBIN, 1979).

Le dosage du glucose, du fructose, du saccharose après hydrolyse, et de l'amidon hydrolysé enzymatiquement en glucose, a été effectué suivant les méthodes de dosage enzymatique BOEHRINGER (BERGMEYER, 1979). Le NADPH formé, résultant par réaction couplée de la transformation du glucose en gluconate-6phosphate, est dosé à $340 \mathrm{~nm}$.

\section{RÉSULTATS}

\section{A. Evolution dest glucides solubles au cours du gros- sissement du tubercule}

\section{Dans le tubercule des plantes témoins}

L'étude des glucides solubles totaux dans le tubercule au cours de son développement permet de distinguer trois phases en relation avec le développement de la partie aérienne (fig. 1) :

- Une phase de faible accumulation qui dure environ 4 semaines après le début de renflement du tubercule et qui se termine à l'apparition des premiers bourgeons floraux (PBF).

- Une phase d'accumulation très rapide qui se poursuit jusqu'au début de remplissage actif des gousses. La courbe représentant les glucides totaux s'infléchit cependant après l'anthèse des premières fleurs (marquées $\mathrm{PG}$ : premières gousses) et cumule à la fin de la formation des gousses. La teneur en glucides solubles totaux s'établit alors entre 30 et 35 p. 100 de la matière sèche.

- Enfin la période de remplissage actif des gousses se traduit dans le tubercule par une légère baisse des glucides solubles totaux qui s'observe jusqu'à la récolte.

Les glucides solubles totaux sont constitués essentiel- 


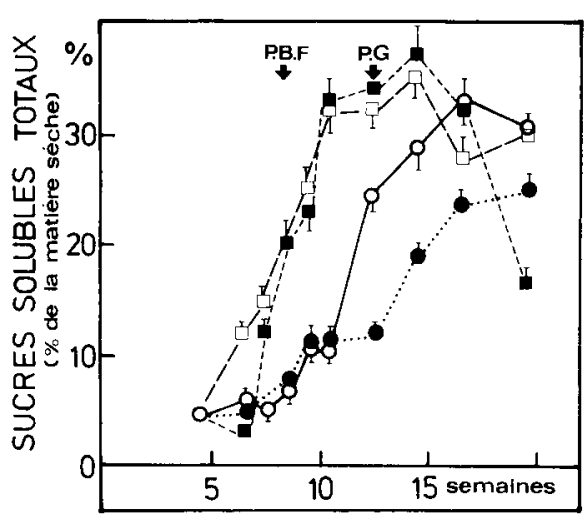

Figure 1

Evolution des sucres solubles totaux dans le tubercule du dolique tubéreux en développement soumis aux traitements suivants : $(0)$

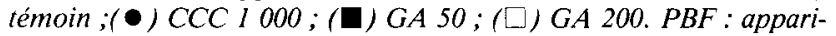
tion des premiers bourgeons floraux; $P G$ : début de formation des premières gousses.

Change in soluble carbohydrates in growing tuberous root of yam bean under different treatments : $(O)$ control ; ( ) CCC I 000 ; (ロ) $G A 50$; ( $\square$ ) $G A$ 200. PBF and $P G$ stand respectively for appearance of first floral buds and beginning of pod set.

lement du glucose, fructose et saccharose. Le fructose est toujours en concentration légèrement supérieure à celle du glucose. Leur évolution reflète celle des sucres solubles totaux. Cependant la teneur en fructose se stabilise rapidement à son maximum, autour de 10 p. 100 de la matière sèche, entre l'anthèse des premières fleurs (PG : premières gousses) et la récolte des tubercules. Le glucose n'atteint son maximum que plus tard, en même temps que les sucres solubles totaux. La teneur en saccharose du tubercule est toujours plus faible que celle en glucose et fructose. Elle s'annule pratiquement dès le début de la formation des gousses (fig. 3A).

\section{Effet de l'acide gibbérellique}

L'acide gibbérellique à la dose de 50 ou 200 ppm favorise une accumulation précoce des sucres solubles totaux, qui débute dès la première application. Le maximum qui est atteint deux semaines plus tôt que chez les plantes témoins (fig. 1) se situe aux environs de 35 p. 100 par rapport à la matière sèche. A la récolte des tubercules on retrouve la même teneur en glucides solubles totaux dans les tubercules des plantes témoins et celles traitées au GA 200. Dans les tubercules des plantes traitées au GA 50 on assiste, au contraire, à un effondrement des sucres solubles totaux pendant la phase de remplissage actif des gousses. A la récolte la teneur n'est plus que de 16 p. 100 au lieu de 30 p. 100 dans les tubercules témoins ou traités au GA 200.

Le glucose et le fructose augmentent très rapidement (fig. 2A et B). Le fructose, ici encore, atteint son maximum plus rapidement que le glucose (fig. 2B). L'effondrement des glucides totaux chez les plantes traitées par GA 50 semble résulter d'une diminution des teneurs du glucose et à un moindre degré du fructose. En 3 semaines de la $16^{\mathrm{e}}$ à la $19^{\mathrm{e}}$, les teneurs en glucose et fructose du tubercule des plantes traitées au GA 50 passent respectivement de 11,5 à $2 \mathrm{p} .100$ et de 12,5 à 7 p. 100 .

L'effet de l'acide gibbérellique porte aussi sur

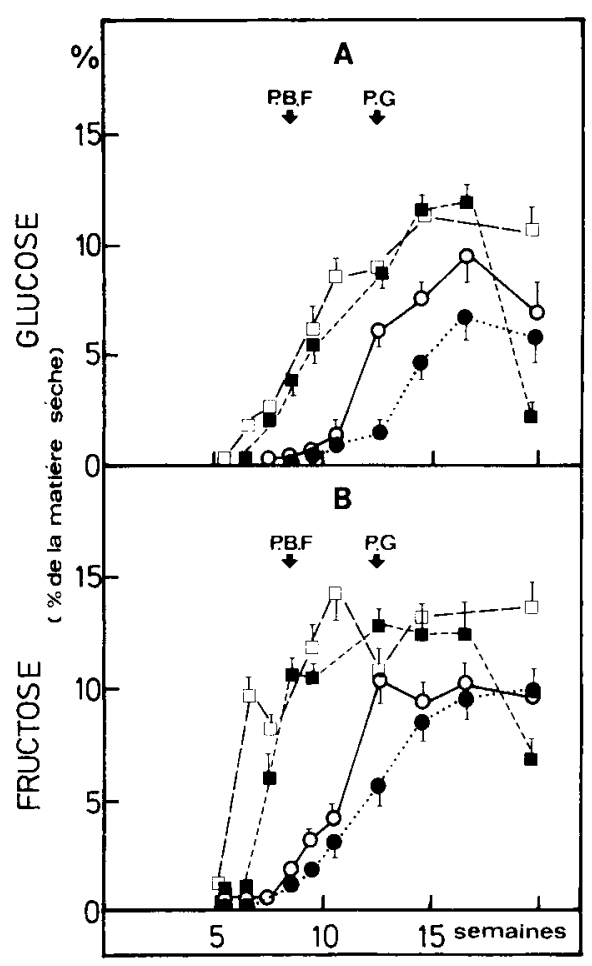

Figure 2

Evolution du glucose et du fructose au cours du développement du tubercule des plantes ayant reçu les traitements suivants : témoin $(O)$, $G A 50(\boldsymbol{\square})$ et $G A 200(\square)$ et CCC $1000(\bullet)$ ). PBF : premiers bourgeons floraux ; $P G$ : début de formation des premières gousses.

Glucose and fructose changes during the development of tuberous root : control (O); GA $50(\square)$ and $G A 200(\square)$ and $C C C 1000(\bullet)$. $P B F$ : appearance of first floral buds; $P G$ : beginning of pod set.

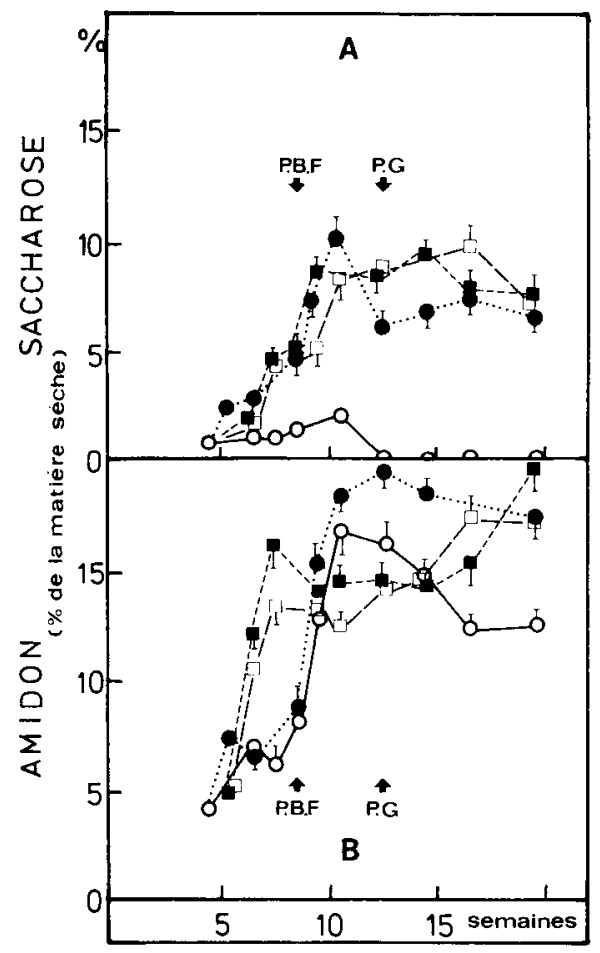

Figure 3

Evolution du saccharose ( $A$ ) et de l'amidon (B) dans le tubercule du dolique tubéreux. GA $50(\mathbf{\square}) ; G A 200(\square)$ et $C C C(\bullet)$ et témoin (O).

Change in sucrose $(A)$ and starch $(B)$ contents in the growing tube-

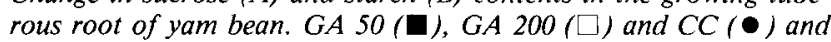
control $(\mathrm{O})$. 
l'accumulation du saccharose. Alors que ce dernier est à peine détectable dans les tubercules des plantes témoins (fig. 3A) après la formation des premiers bourgeons floraux, il s'accumule au contraire dans les tubercules des plantes traitées à l'acide gibbérellique avec une teneur qui fluctue entre 7 et 10 p. 100 pendant toute la floraison et le remplissage des gousses dans la partie aérienne. Cette accumulation ne semble pas s'opérer au détriment du glucose et du fructose dont on peut, au premier examen, supposer qu'ils dérivent de l'hydrolyse du saccharose.

\section{Effet du chlorure de chlorocholine}

L'effet du CCC sur les glucides solubles totaux (fig. 1) se manifeste par une inhibition partielle de l'accumulation de ces sucres. Cet effet, inexistant jusqu'à la $10^{\mathrm{e}}$ semaine est surtout manifeste après l'apparition des premiers bourgeons floraux et se maintient jusqu'à la récolte du tubercule. Vers la $12^{\mathrm{e}}$ semaine, la teneur en glucides totaux des tubercules des plantes témoins est 2 fois plus élevée que celle rencontrée dans les tubercules des plantes traitées au CCC.

En comparant l'effet du CCC sur le glucose et le fructose (fig. $2 \mathrm{~A}$ et $\mathrm{B}$ ), on peut remarquer que la teneur en ce dernier sucre est la moins affectée. En outre le décalage entre les teneurs, constaté entre les plantes traitées et les témoins, est rattrapé au moment de la récolte, ce qui suppose la diminution progressive de l'effet du CCC après l'arrêt des traitements.

Le CCC provoque aussi l'accumulation du saccharose dans les tubercules. Jusqu'à la $10^{\mathrm{e}}$ semaine (fig. 3A) aucune différence ne s'observe entre les plantes traitées au GA et au CCC. Tout au long de la période de formation et du remplissage des gousses, le niveau du saccharose dans le tubercule, qui se situe autour de 6,5 p. 100, n'est pas affecté.

Le GA et le CCC, aux doses employées, ont le même effet en ce qui concerne l'accumulation du saccharose alors que leurs effets sont opposés sur le glucose et le fructose.

\section{B. Effet des facteurs de croissance sur l'amidon du tubercule}

La teneur maximale en amidon des tubercules chez les plantes témoins est atteinte vers la $10^{\mathrm{e}}$ semaine située entre l'apparition des premiers bourgeons floraux et le début de la formation des gousses. Elle décrô̂t progressivement pendant la formation et le remplissage des gousses (fig. 3B).

Les facteurs de croissance modifient ce schéma d'évolution. L'acide gibbérellique, quelle que soit la dose utilisée, provoque une accumulation rapide de l'amidon avec un pic vers la $8^{\mathrm{e}}$ semaine. Il atteint respectivement 13,5 p. 100 et 16 p. 100 de la matière sèche du tubercule avec GA 200 et GA 50. Sa teneur se stabilise pratiquement jusqu'à la $16^{\mathrm{e}}$ semaine, pendant la phase de formation et de remplissage des gousses. La vitesse d'accumulation de l'amidon est alors proportionnelle à celle du stockage de la matière sèche dans le tubercule. Une réduction de la demande en assimilats des gousses entraîne une nouvelle augmentation de la teneur en amidon qui atteint 20 p. 100 pour GA et 17 p. 100 pour GA 200 à la récolte.
L'effet du CCC ne s'observe qu'après la $11^{\mathrm{e}}$ semaine. Il se traduit par une élévation de la teneur en amidon qui passe par un maximum deux semaines après celui du tubercule des plantes témoins. La chute du niveau d'amidon rencontrée chez les plantes témoins a été inhibée par l'application du CCC. A la récolte il se situe autour de 17,5 p. 100 pour les plantes traitées et 12,5 p. 100 pour les témoins.

\section{DISCUSSION}

De l'étude de la composition glucidique du tubercule de $P$. erosus nous retenons :

- L'enrichissement en sucres solubles totaux du tubercule chez les plantes ayant reçu des applications de l'acide gibbérellique. L'effet inverse est obtenu en présence du chlorure de chlorocholine.

- L'accumulation du saccharose dans les tubercules dont les plantes sont traitées avec l'un ou l'autre des facteurs de croissance.

- L'activation de la synthèse et l'accumulation de l'amidon dès la première application du GA. L'effet du CCC ne s'observe que plus tard, pendant la phase finale du remplissage des gousses et du tubercule où il inhibe la chute de la teneur en amidon constatée dans les tubercules des plantes témoins.

L'enrichissement des tubercules en sucres solubles totaux notamment en sucres réducteurs a été rapporté par LOVELL \& BOOTH (1967) sur la pomme de terre ayant reçu une application foliaire de GA, et par MARES et al. (1981) sur des tubercules dont le stolon avait été localement traité par ce facteur de croissance. Les auteurs expliquent cet effet par l'activation de l'invertase qui hydrolyse le saccharose, bloquant ainsi la conversion de ce dernier en amidon et l'appel du puits « tubercule ». Le CCC antagoniste du GA interviendrait en sens inverse pour favoriser sa transformation en amidon et pour inhiber son hydrolyse en sucres réducteurs.

Cette hypothèse ne permet pas d'expliquer de façon satisfaisante les résultats trouvés dans le tubercule de Pachyrhizus. En effet, l'application du GA au niveau de la partie aérienne entraîne non seulement un enrichissement en saccharose mais aussi une activation de la synthèse de l'amidon et son accumulation dans le tubercule sans affecter le niveau du disaccharide. Le CCC exerce le même effet en élevant la teneur du saccharose et celle de l'amidon.

L'accumulation de saccharose et l'amélioration de la teneur en amidon en présence de deux facteurs de croissance qui n'ont pas habituellement les mêmes effets peuvent s'expliquer par leur action sur la partie aérienne. Elle serait liée dès l'application, chez les plantes traitées au GA, à l'activation de la photosynthèse et à une distribution des assimilats à la fois au tubercule et à la partie aérienne contribuant ainsi à la mise en place d'un appareil à capacité photosynthétique accrue. A l'apparition du second puits constitué par les gousses en formation, la taille de la source a été suffisante pour couvrir ses besoins et en même temps assurer le flux d'assimilats nécessaires à l'accumulation du saccharose et au maintien du niveau d'amidon. Ceci expliquerait aussi l'absence de compétition entre 
les deux puits de réserve qui s'est traduite, dans le tubercule des plantes témoins, par une disparition du saccharose et la chute de la teneur en amidon pendant la phase de formation et de remplissage des gousses. Une réduction de la demande des gousses pendant la phase précédant la récolte a provoqué une vitesse de synthèse d'amidon supérieure à celle du grossissement du tubercule en présence de GA 50 et un redémarrage de la croissance de la partie aérienne avec GA 200. Pendant cette dernière période la chute du glucose et du fructose dans le tubercule des plantes traitées au GA 50 est contemporaine de l'activation de l'accumulation de l'amidon, ce qui supposerait leur transformation partielle en ce polysaccharide.

En présence du CCC, l'accumulation du saccharose est aussi rapide puisqu'il s'observe dès l'application. $\mathrm{Au}$ niveau de la teneur en amidon l'effet n'est perçu qu'au moment où s'instaure la compétition entre les deux puits de réserve. Ici encore, il ne peut être compris qu'en évoquant une modification non de la taille de la source comme en présence du GA mais de son fonctionnement. En effet, les résultats de PROKHORCHIK \& MASHTOKOV (1972 cités par EL-ABD et al., 1980) montrent que les doses moyennes de CCC augmentent la teneur en chlorophylles $\mathbf{a}$ et $\mathbf{b}$ et en caroténoïdes, l'intensité photosynthétique et la phosphorylation cyclique, donc tous les facteurs susceptibles d'élever le pool des assimilats.

Si l'on tient compte à la fois de la matière sèche produite par plante et par tubercule (ZINSOU et al., 1987) et la teneur finale en amidon à la récolte sous les différents traitements, les rendements en amidon par plante sont $14 \mathrm{~g}, 15 \mathrm{~g}, 35 \mathrm{~g}$ et $57 \mathrm{~g}$ respectivement pour le témoin, CCC 1000, GA 200 et GA 50. Le traitement à l'acide gibbérellique améliore le rendement en amidon chez le dolique tubéreux qui se distingue ainsi des autres plantes à tubercules.

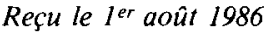
Accepté le 5 juillet 1987.

\section{RÉFÉRENCES BIBLIOGRAPHIQUES}

Bergmeyer H. V., 1979. Principes de l'analyse enzymatique. Technique et documentation, Paris, $303 \mathrm{p}$.

Cerning-Beroard J., 1975. The use of invertase for determination of sucrose. Application to cereals, cereal products and other plant materials. Cereal chem., 52, 431-438.

Fl-Abd S. O., El-Beltagy M. S., Abou-Hussein M. R., El-Beltagy A. S., Maksoud M. A., 1980. Effect of some growth regulators on growth and development of potato plants. Egypt. J. Hort., 7, 169180 .

Lovell P. H., Booth A., 1967. Effects of gibberellic acid on growth, tuber formation and carbohydrate distribution in Solanum. New Phytol., 525-537.

Mares D. J., Marschner H., Krauss A., 1981. Effect of gibberellic acid on growth and carbohydrate metabolism of developing tubers of potato (Solanum tuberosum). Physiol. Plant., 52, 267-274.

Marta Evans I., Boulter D., Eaglesham A. R. J., Dart P. J., 1977. Protein content and protein quality of tuberous roots of some legumes determined by chemical method. Qual. Plant. PI. Fds. Hum. Nutr., 27, 275-285.
Martinez M., 1959. Las plantas utiles de la flora mexicana. Ed. Botas. Mex. 340-344.

Schroeder C. A., 1967. The jicama, a rootcrop from Mexico. University of California, 65-71.

Srivastava G. S., Shukla D. S., Awaasthi D. N., 1973. We can grow sankalu in the plains of Uttar Pradesh. Indian Farming, 33, 3-9.

Thivend P., Mercier C., Guilbot A., 1965. Dosage de l'amidon dans les milieux complexes. Ann. Biol. anim. Bioch. Biophys., 5, 513526.

Tollier M. T., Robin J. P., 1979. Adaptation de la méthode à l'orcinol sulfurique au dosage automatique des glucides neutres totaux : conditions d'application aux extraits d'origine végétale. Ann. Technol. agri., 28, 1-15.

Trebos 1., 1982. Détermination de la composition glucidique du tubercule de Pachyrhizus erosus. Mémoire IUT-CRAAG, 80 pages.

Zinsou C., Venthou-Dumaine A., Vansuyt G., 1987. Croissance et développement du Pachyrhizus erosus Urban. 1. Effets de l'acide gibbérellique et du chlorure de chlorocholine en jours courts. Agronomie, 7 (9), 677-683. 